\title{
Veronicastrum axillare Alleviates Lipopolysaccharide-Induced Acute Lung Injury via Suppression of Proinflammatory Mediators and Downregulation of the NF- $\kappa$ B Signaling Pathway
}

\author{
Quanxin Ma, ${ }^{1}$ Kai Wang, ${ }^{2,3}$ Qinqin Yang, ${ }^{4}$ Shun Ping, ${ }^{2}$ Weichun Zhao, ${ }^{5}$ \\ Qiyang Shou, ${ }^{1}$ Weimin Zhou, ${ }^{1}$ and Minli Chen ${ }^{1}$ \\ ${ }^{1}$ Animal Experimental Research Center, Zhejiang Chinese Medical University, Hangzhou 310053, China \\ ${ }^{2}$ College of Animal Sciences, Zhejiang University, Hangzhou 310058, China \\ ${ }^{3}$ Institute of Apicultural Research, Chinese Academy of Agricultural Sciences, Beijing 100093, China \\ ${ }^{4}$ Zhejiang Institute of Traditional Chinese Medicine, Hangzhou 310007, China \\ ${ }^{5}$ College of Bioengineering, Zhejiang Chinese Medical University, Hangzhou 310053, China
}

Correspondence should be addressed to Minli Chen; cmli991@aliyun.com

Received 18 August 2016; Accepted 28 September 2016

Academic Editor: Jie Yin

Copyright (C) 2016 Quanxin Ma et al. This is an open access article distributed under the Creative Commons Attribution License, which permits unrestricted use, distribution, and reproduction in any medium, provided the original work is properly cited.

\begin{abstract}
Veronicastrum axillare is a traditional medical plant in China which is widely used in folk medicine due to its versatile biological activities, especially for its anti-inflammatory effects. However, the detailed mechanism underlying this action is not clear. Here, we studied the protective effects of V. axillare against acute lung injury (ALI), and we further explored the pharmacological mechanisms of this action. We found that pretreatment with $V$. axillare suppressed the release of proinflammatory cytokines in the serum of ALI mice. Histological analysis of lung tissue demonstrated that $V$. axillare inhibited LPS-induced lung injury, improved lung morphology, and reduced the activation of nuclear factor $-\kappa \mathrm{B}(\mathrm{NF}-\kappa \mathrm{B})$ in the lungs. Furthermore, the anti-inflammatory actions of $V$. axillare were investigated in vitro. We observed that $V$. axillare suppressed the mRNA expression of interleukin- $1 \beta$ (IL- $1 \beta$ ), IL-6, monocyte chemotactic protein-1 (MCP-1), cyclooxygenase-2 (COX-2), and tumor necrosis factor- $\alpha$ (TNF- $\alpha$ ) in RAW264.7 cells challenged with LPS. Furthermore, pretreatment of $V$. axillare in vitro reduced the phosphorylation of p65 and I $\kappa \mathrm{B}-\alpha$ which is activated by LPS. In conclusion, our data firstly demonstrated that the anti-inflammatory effects of $V$. axillare against ALI were achieved through downregulation of the NF- $\kappa \mathrm{B}$ signaling pathway, thereby reducing the production of inflammatory mediators.
\end{abstract}

\section{Introduction}

Acute lung injury (ALI) is a prototypical inflammatory disease. ALI's most significant pathological feature is the acute lung inflammation, including inflammatory cell recruitment and the release of proinflammatory mediators $[1,2]$. Lipopolysaccharide (LPS) is the major component of the cell wall in Gram-negative bacteria. LPS can be used to produce a classical animal model of ALI associated with the activation of monocytes, overflow of pulmonary neutrophils, increased levels of alveolar-capillary permeability, and diffuse alveolar damage [3]. During the initiation of the host defense process, LPS is recognized by Toll-like receptor 4 (TLR4), which then activates several intracellular signaling pathways, among which nuclear factor- $\kappa \mathrm{B}(\mathrm{NF}-\kappa \mathrm{B})$ pathway is the predominant one. The activation of NF- $\kappa \mathrm{B}$ eventually leads to the upregulation of several inflammatory factors [4]. Therefore, interfering with NF- $\kappa \mathrm{B}$ signaling pathway is a promising therapeutic regimen for the treatment of acute inflammatory disease, like ALI.

Veronicastrum axillare is a folk medicine which is widely used in many regions in East Asia to treat ascites, burns, and snakebites, among other ailments [5]. Our previous work indicated that $V$. axillare significantly inhibits ethanol-induced gastric ulcers [6]. Recently, it was shown that the ethyl acetate (EtOAc) extracts of $V$. axillare displayed potent antiinflammatory activity in vivo using xylene-induced mouse ear edema model [7]. Nevertheless, effects of $V$. axillare 
against ALI and potential anti-inflammatory mechanisms are poorly understood. Here, we assessed the anti-inflammatory effects of $V$. axillare in vivo employing LPS-induced ALI mice. We also explored the underlying mechanisms of this action, including the possible inhibition on the activation of macrophages.

\section{Materials and Methods}

2.1. Animals and Chemical Reagents. Male ICR mice $(20 \pm$ $2 \mathrm{~g})$ were purchased from the Shanghai Laboratory Animal Research Center and housed in the Animal Experimental Center, Zhejiang Chinese Medical University. Four mice in one cage were housed in a room with controlled lighting $(12 \mathrm{~h}$ light/dark cycle) and temperature $\left(20 \pm 2^{\circ} \mathrm{C}\right)$, and the air was filtered by a ventilation system with relative humidity of $50 \%$. Mice were fed with a standard laboratory diet (AIN93 formulation for experimental animals, Xietong Biotechnology, Nanjing, China) and water ad libitum and received careful care to fulfill the requirement of animal welfare during experimental periods. The animal experimental protocols described in this study were in accordance with the regulations from the Animal Experimental Center, Zhejiang Chinese Medical University, and were approved by Zhejiang Chinese Medicine University Animal Care Committee.

LPS (Escherichia coli O111:B4) and alkaline phosphataseconjugated secondary antibody (anti-rabbit IgG) were purchased from Sigma (St. Louis, MO, USA). Primary rabbit monoclonal antibodies against phospho-I $\kappa \mathrm{B}-\alpha(\mathrm{pS} 36), \mathrm{I} \kappa \mathrm{B}-$ $\alpha$, and $\beta$-tubulin were purchased from Epitomics (Burlingame, CA, USA). Primary rabbit monoclonal antibodies phospho-NF- $\kappa$ B p65 (Ser536) were purchased from Cell Signaling (Beverly, MA, USA). All the other reagents were obtained from Sangon Biotechnology (Shanghai, China) unless indicated specifically in each section.

2.2. Preparation of $V$. axillare Aqueous Extract. Whole $V$. axillare plants were gathered in Lishui, Zhejiang Province, China, and identified by Professor Zhensheng Yao (School of Pharmacy, Zhejiang Chinese Medical University, China). The aqueous extract of $V$. axillare was prepared from air-dried and powdered whole plants. In brief, the powdered plants were soaked for $0.5 \mathrm{~h}$ in $20^{\circ} \mathrm{C}$ with $1 \mathrm{~L}$ water. The samples were then boiled for $1.5 \mathrm{~h}$ with gentle heat three times in $100^{\circ} \mathrm{C}$. The decoction was then concentrated to $140 \mathrm{~g} / \mathrm{L}$ for storage at $4^{\circ} \mathrm{C}$ in the fridge in the dark. The extract was diluted to the corresponding concentration with pure water before use [6].

2.3. Experimental Protocols for the Animal Studies. After $4 \mathrm{~d}$ of adaptation, the mice were randomly divided into five groups $(n=8)$ as follows: (1) control group (normal saline, $10 \mathrm{~mL} / \mathrm{kg}$ orally); (2) LPS-induced model group (LPS, $1 \mathrm{mg} / \mathrm{kg}$ via tail vein injection); (3) $V$. axillare low-dose group ( $V$. axillare, $300 \mathrm{mg} / \mathrm{kg}$ orally and LPS injection); (4) V. axillare high-dose group ( V. axillare, $1200 \mathrm{mg} / \mathrm{kg}$ orally and LPS injection); and (5) dexamethasone-treated group (DEX and LPS injection). The mice were orally pretreated with $V$. axillare (300 or $1200 \mathrm{mg} / \mathrm{kg}$ ) for 3 consecutive days, while mice from other groups (control, LPS, and DEX group) received the normal saline solutions. The mice in the DEX-treated group were given DEX orally $(2 \mathrm{mg} / \mathrm{kg}) 1 \mathrm{~h}$ before LPS challenge. One hour after the final $V$. axillare treatment, all animals except for the control group were challenged with LPS via tail vein to induce ALI. The mice were sacrificed $6 \mathrm{~h}$ after the LPS tail injection [8]. Blood samples were collected and centrifuged at $3000 \mathrm{rpm}$ for $10 \mathrm{~min}$ at $4^{\circ} \mathrm{C}$ to obtain serum, which was then stored at $-80^{\circ} \mathrm{C}$ for further experiments. Lung tissues were harvested simultaneously from mice and soaked in $10 \%$ formalin.

2.4. Histological Evaluation of the Lungs. Mice lung tissues were immediately dehydrated and embedded in paraffin. Paraffin sections ( $4 \mu \mathrm{m}, 6-10$ per lung) were sliced and stained with hematoxylin and eosin (H\&E) using a standard conventional method. Pathological sections were observed under a light microscope at 400x amplification. Photos were taken by the attached camera (Nikon).

Immunostaining was performed according to Lin's experimental method [9]. The sections were blocked with BSA (Sangon, Shanghai) and then incubated with NF- $\kappa \mathrm{B}$ primary antibody (Santa Cruz) overnight at $4^{\circ} \mathrm{C}$. Horseradish peroxidase- (HRP-) conjugated secondary antibodies were used for $1 \mathrm{~h}$ incubation. Finally, the sections were treated with Dolichos biflorus agglutinin (DBA) as a color reagent and counterstained with hematoxylin. The sections were viewed with the light microscope, and the pathological pictures were analyzed by ImageJ software.

2.5. Cell Culture and Cell Viability Assay. The murine macrophage RAW264.7 cell line was donated by Professor Fuliang $\mathrm{Hu}$ (College of Animal Sciences, Zhejiang University, China). The cells were cultured in DMEM supplemented with $10 \%$ fetal bovine serum (FBS), $100 \mathrm{U} / \mathrm{mL}$ penicillin, and $100 \mathrm{~g} / \mathrm{mL}$ streptomycin at $37^{\circ} \mathrm{C}$ in $5 \% \mathrm{CO}_{2}$ atmosphere. The viability of RAW264.7 cells incubated with $V$. axillare was determined by using cell counting kit-8 (CCK-8, Dojindo, Japan). RAW264.7 cells were seeded in 96-well plates at a density of $10^{4}$ cells/well. After incubating for $24 \mathrm{~h}$, the cells were treated with various concentrations of $V$. axillare extract for an additional $24 \mathrm{~h}$. CCK- 8 reagent $(10 \mu \mathrm{L})$ was added to the cells and then further incubated for $2 \mathrm{~h}$. The OD values of each well were measured using a microplate reader at $450 \mathrm{~nm}$ (Bio-Rad Model 550, CA).

2.6. Measurement of Inflammatory Cytokines. Levels of various inflammatory cytokines, including interleukin-6 (IL-6), IL-10, monocyte chemotactic protein-1 (MCP-1), interferon$\gamma($ IFN- $\gamma$ ), tumor necrosis factor- $\alpha$ (TNF- $\alpha$ ), and IL-12p70, were assessed using a cytometric bead array (CBA) mouse inflammation kit (BD Biosciences). Serum samples were diluted 25 -fold as instructed by the manufacturer.

2.7. RNA Extraction and Quantitative Real-Time PCR ( $q P C R)$. Total cellular RNA was extracted using an RNA extraction kit (Aidlab Biotechnologies, Beijing, China) following the 
TABLE 1: Primers used for qRT-PCR experiments.

\begin{tabular}{|c|c|c|}
\hline Gene & Primer sequence & GenBank accession number \\
\hline \multirow{2}{*}{ IL- $1 \beta$} & $5^{\prime}$-CCAACAAGTGATATTCTCCATGAG-3' & \multirow{2}{*}{ NM_008361.3 } \\
\hline & 5'-ACTCTGCAGACTCAAACTCCA-3' & \\
\hline \multirow{2}{*}{ IL-6 } & $5^{\prime}$-CTCTGCAAGAGACTTCCATCC-3' & \multirow{2}{*}{ NM_031168.1 } \\
\hline & $5^{\prime}$-GAATTGCCATTGCACAACTC-3' & \\
\hline \multirow{2}{*}{ IL-10 } & $5^{\prime}$-CTATGCTGCCTGCTCTTACTG-3' & \multirow{2}{*}{ NM_010548.2 } \\
\hline & $5^{\prime}$-CAACCCAAGTAACCCTTAAAGTC-3' & \\
\hline \multirow{2}{*}{ TNF- $\alpha$} & 5'-CCACGCTCTTCTGTCTACTG-3' & \multirow{2}{*}{ NM_013693.2 } \\
\hline & $5^{\prime}$-ACTTGGTGGTTTGCTACGAC-3' & \\
\hline \multirow{2}{*}{ MCP-1 } & 5'-AAGAAGCTGTAGTTTTTGTCACCA-3' & \multirow{2}{*}{ NM_011333.3 } \\
\hline & $5^{\prime}$-TGAAGACCTTAGGGCAGATGC-3' & \\
\hline \multirow{2}{*}{$\mathrm{COX}-2$} & $5^{\prime}$-GAAATATCAGGTCATTGGTGGAG-3' & \multirow{2}{*}{ NM_011198.3 } \\
\hline & 5'-GTTTGGAATAGTTGCTCATCAC-3' & \\
\hline \multirow{2}{*}{ GAPDH } & $5^{\prime}$-GAGAAACCTGCCAAGTATGATGAC-3' & \multirow{2}{*}{ NM_008084.2 } \\
\hline & $5^{\prime}$-TAGCCGTATTCATTGTCATACCAG-3' & \\
\hline
\end{tabular}

TABLE 2: Effect of $V$. axillare on serum inflammatory cytokines in LPS-challenged mice ${ }^{\mathrm{a}}$.

\begin{tabular}{lccccccc}
\hline Group & Dose $(\mathrm{mg} / \mathrm{kg})$ & $\mathrm{MCP}-1(\mathrm{ng} / \mathrm{mL})$ & $\mathrm{IFN}-\gamma(\mathrm{ng} / \mathrm{mL})$ & $\mathrm{TNF}-\alpha(\mathrm{pg} / \mathrm{mL})$ & $\mathrm{IL}-6(\mathrm{ng} / \mathrm{mL})$ & $\mathrm{IL}-10(\mathrm{pg} / \mathrm{mL})$ & $\mathrm{IL}-12 \mathrm{p} 70(\mathrm{pg} / \mathrm{mL})$ \\
\hline Control & - & $\mathrm{ND}$ & $\mathrm{ND}$ & $\mathrm{ND}$ & $\mathrm{ND}$ & $\mathrm{ND}$ & $\mathrm{ND}$ \\
Model & - & $98.27 \pm 10.69$ & $5.22 \pm 0.31$ & $1897.57 \pm 93.31$ & $96.00 \pm 10.66$ & $237.15 \pm 2.04$ & $100.60 \pm 8.08$ \\
$V$. axillare & 300 & $81.31 \pm 6.52^{*}$ & $6.44 \pm 0.62$ & $1290.10 \pm 250.03^{*}$ & $83.17 \pm 8.17$ & $225.95 \pm 9.78$ & $63.72 \pm 2.57^{*}$ \\
$V$. axillare & 1200 & $60.45 \pm 2.42^{* *}$ & $3.02 \pm 0.09^{* *}$ & $711.36 \pm 32.64^{* *}$ & $33.82 \pm 4.47^{* *}$ & $118.84 \pm 7.28^{* *}$ & $25.15 \pm 2.04^{*}$ \\
Dexamethasone & 2 & $67.93 \pm 1.18^{* *}$ & $2.69 \pm 0.64^{* *}$ & $850.81 \pm 58.44^{* *}$ & $52.05 \pm 7.37^{* *}$ & $180.21 \pm 11.53^{* *}$ & $26.96 \pm 0.52^{* *}$ \\
\hline${ }^{a}$ Values are the means $+\mathrm{SD}\left(n=8\right.$ for each group) ${ }^{*} p<0.05$ and $^{* *} p<0.01$ indicate a significant difference compared to the model group. ND: not detected
\end{tabular}

directions of the manufacturer. For each sample, cDNA was synthesized using the Prime Script RT reagent kit (TaKaRa, Dalian, China). The primer sequences are shown in Table 1 and were synthesized by Sangon Biotechnology Co., Ltd. qPCR was executed in an automated thermal cycler (Eppendorf, Hamburg, Germany) in a final volume of $25 \mu \mathrm{L}(2 \mu \mathrm{L}$ cDNA, $12.5 \mu \mathrm{L} \mathrm{SYBR}{ }^{\circledR}$ Premix Ex Taq, $1 \mu \mathrm{L}$ of each primer, $10 \mu \mathrm{mol} / \mathrm{L}$, and $8.5 \mu \mathrm{L} \mathrm{ddH}_{2} \mathrm{O}$ ). The cycling reaction was performed using a standard two-step PCR reaction.

2.8. Western Blotting Analysis. For western blotting analysis, cells were washed twice with chilled PBS and then lysed in lysis buffer (50 mM Tris, pH 7.5, $150 \mathrm{mM} \mathrm{NaCl}, 0.5 \%$ NP40, 10\% glycerol, $2 \mathrm{mM}$ DTT, $1 \mathrm{mM}$ leupeptin, and $1 \mathrm{mM}$ PMSF) on ice for $30 \mathrm{~min}$. Obtained cell lysates were cleared by centrifugation at $12,000 \times \mathrm{g}$ for $15 \mathrm{~min}$ at $4^{\circ} \mathrm{C}$. Cellular proteins $(30 \mu \mathrm{g})$ were then mixed with sampling buffer and further boiled for $5 \mathrm{~min}$ at $95^{\circ} \mathrm{C}$. Then, proteins $(10 \mu \mathrm{g})$ were separated by SDS-PAGE gels and then transferred to polyvinylidene fluoride (PVDF) membranes. The membranes were blocked with $5 \%$ nonfat milk at room temperature and then probed with primary polyclonal antibodies overnight at $4^{\circ} \mathrm{C}$. After that, the membranes were washed with TBST and then incubated with secondary antibody for $1 \mathrm{~h}$ at room temperature. Then, the membranes were washed again three times in TBST. Finally, the immune reactive protein bands on the membrane were visualized using $10 \mathrm{~mL}$ alkaline phosphatase in a color development buffer.
2.9. Statistical Analysis. All values were presented as the mean \pm SD for at least three independently performed experiments. The data's statistical comparison was performed by one-way analysis of variance (ANOVA), followed by the $t$ test. Statistical significance was set at $p<0.05$. All statistical tests were performed using SPSS 19.0.

\section{Results}

3.1. V. axillare Reduced LPS-Induced Augmentation of Serum Cytokines and Attenuated the Pathological Injuries in Mouse Lungs. In order to assess the protective effect of $V$. axillare in mice with ALI, serum inflammatory cytokines and pulmonary histopathology were evaluated. V. axillare (300 or $1200 \mathrm{mg} / \mathrm{mL}$ ) was administered intragastrically 3 days before LPS injection. As demonstrated in Table 2, LPS stimulation increased inflammatory cytokine production, including IL6 , MCP-1, TNF- $\alpha$, and IL-12p70 in mouse serum. However, the following pretreatment with $V$. axillare $(1200 \mathrm{mg} / \mathrm{kg})$ and $\operatorname{DEX}(2 \mathrm{mg} / \mathrm{kg})$ dramatically inhibited the release of inflammatory cytokines in ALI mice.

After staining with $\mathrm{H} \& \mathrm{E}$, histopathological changes in the lungs of each group were observed. A lot of apparent structural damage was found in the pulmonary tissue from the mice of LPS-treated model group, including increased alveolar wall thickness, inflammatory cell aggregation, and pulmonary hemorrhage (Figure 1). These injuries were not apparent in the control group. Pretreatment with $1200 \mathrm{mg} / \mathrm{kg}$ 


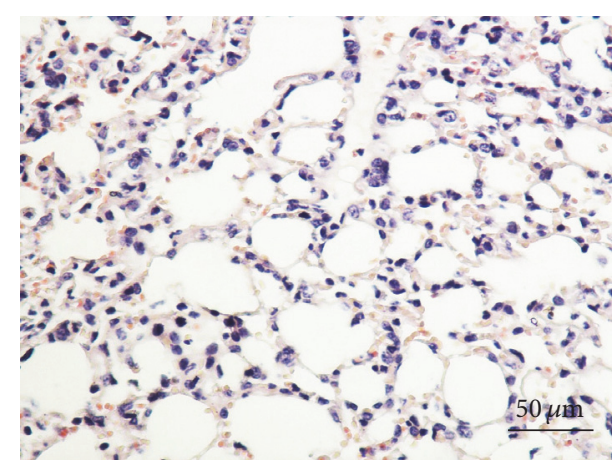

(a)

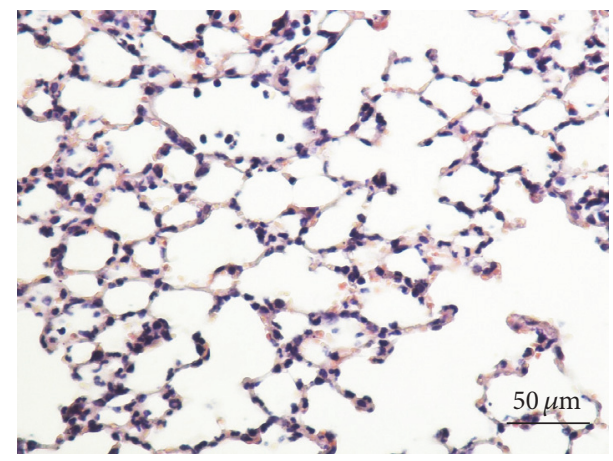

(c)

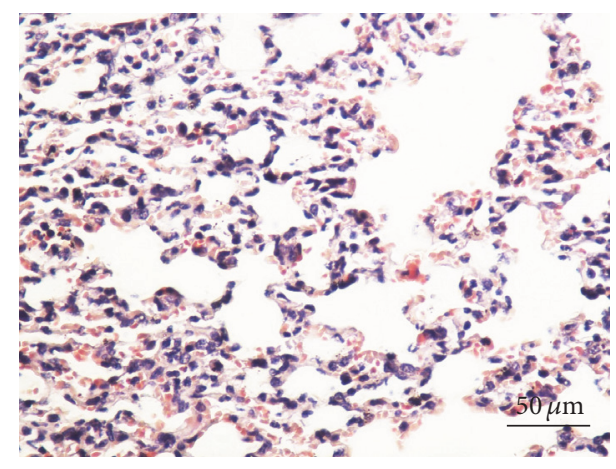

(b)

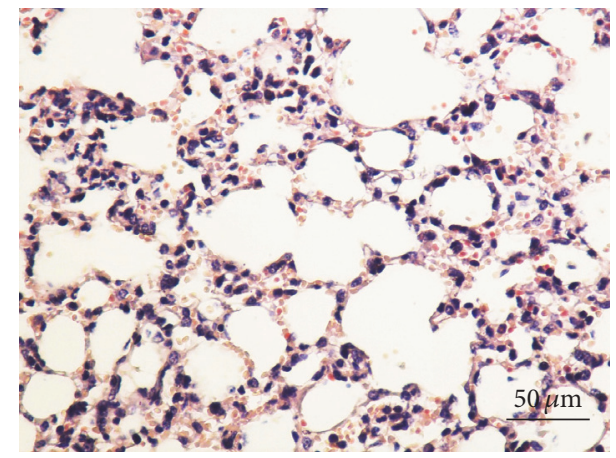

(d)

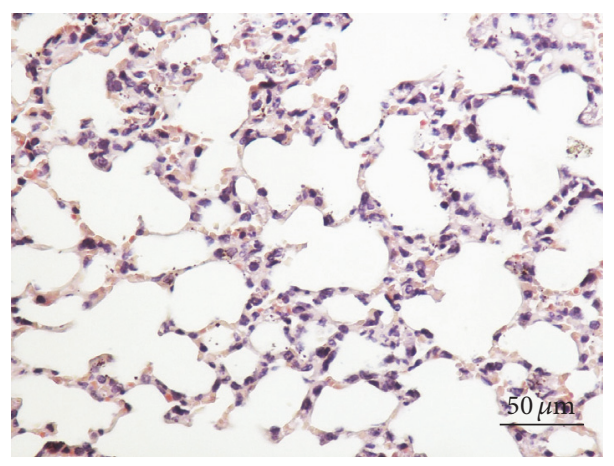

(e)

FIGURE 1: Effects of $V$. axillare on histopathological changes in lung tissues in LPS-induced ALI mice. Representative lung sections from (a) control group; (b) LPS-induced ALI model group; (c) mice injected with LPS and treated with DEX (2 mg/kg); (d and e) mice injected with LPS and given $V$. axillare ( $400 \mathrm{or} 1200 \mathrm{mg} / \mathrm{kg}$ ) are shown. Histological changes were evaluated by H\&E staining and microscopy (original magnification: 200x, scale bar: $50 \mu \mathrm{m}$ ).

V. axillare and $2 \mathrm{mg} / \mathrm{kg}$ DEX significantly inhibited LPSinduced histological changes. These results indicated that $V$. axillare protected against LPS-induced lung tissue damage.

We further evaluated NF- $\kappa \mathrm{B}$ activation in the lung sections using immunohistochemistry. The NF- $\kappa \mathrm{B}$ p65 subunit was stained brown and was primarily localized in the nuclei of inflammatory cells (Figure 2). The expression of NF- $\kappa \mathrm{B}$ in the LPS-treated model group was higher than in the control group. Both $V$. axillare and DEX treatment reduced the positive percentage and density of the NF- $\kappa \mathrm{B}$ p 65 subunit significantly $(p<0.05)$, indicating that both $V$. axillare and
DEX may have inhibited the activation of $\mathrm{NF}-\kappa \mathrm{B}$ in the lungs.

3.2. Effects of $V$. axillare on RAW264.7 Cell Viability. In order to observe whether $V$. axillare had any toxic effects on cells and to find a suitable concentration for application in the subsequent in vitro experiments, we examined the effects of $V$. axillare on cell viability in RAW264.7 cells using a CCK8 assay. Figure 3 shows that there were no cytotoxic effects in RAW264.7 cells treated with $V$. axillare within proper concentrations (up to $900 \mu \mathrm{g} / \mathrm{mL}$ ). Based on these results, we 


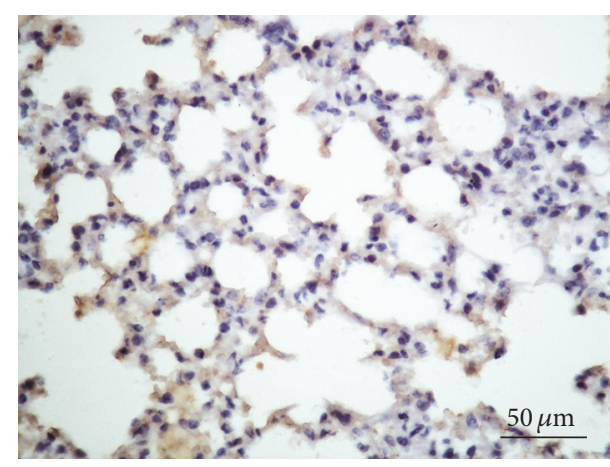

(a)

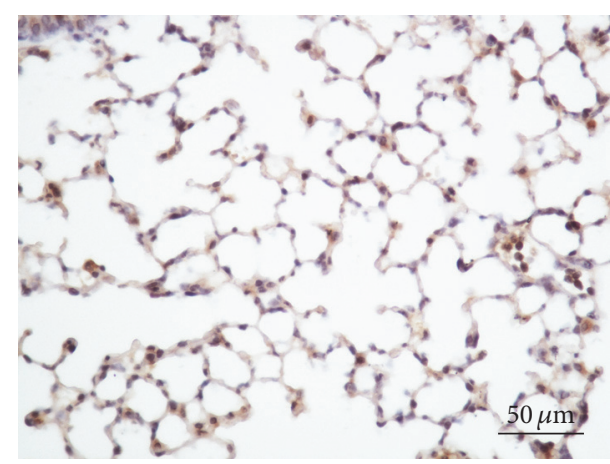

(c)

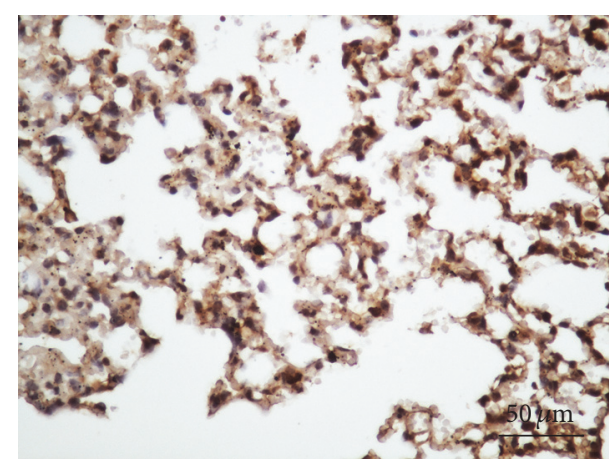

(b)

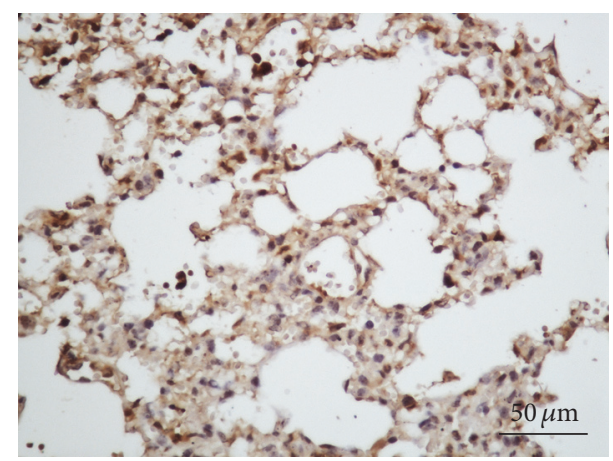

(d)

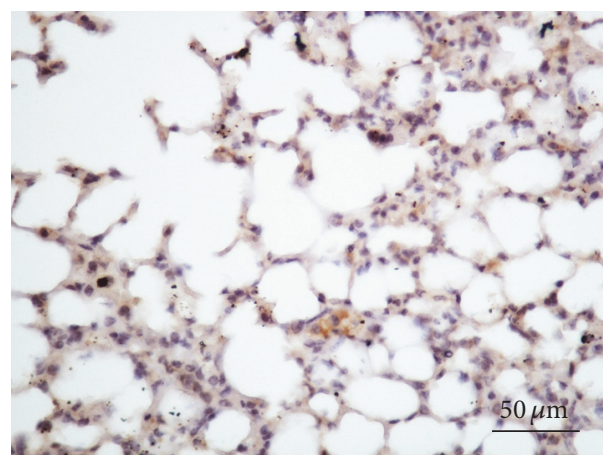

(e)

FIGURE 2: Representative immunohistochemical analysis of NF- $\kappa$ B from (a) the control group, (b) the LPS-treated model group, (c) mice treated with DEX (2 mg/kg), and ( $\mathrm{d}$ and e) mice treated with V. axillare (400 or $1200 \mathrm{mg} / \mathrm{kg})$. Magnification: $200 \mathrm{x}, \mathrm{scale}$ bar: $50 \mu \mathrm{m}$.

selected suitable concentration ranges during the following in vitro experiments.

3.3. V. axillare Modulated Several Key Inflammatory-Related Gene Expressions in LPS-Stimulated RAW264.7 Macrophages. To assess the effect of $V$. axillare on inflammatory mRNA expression in LPS-stimulated RAW264.7 cells, we analyzed the levels of several inflammatory genes (IL- $1 \beta$, IL- 6 , TNF- $\alpha$, MCP-1, and COX-2) through real-time PCR. The RAW264.7 cells were stimulated with LPS alone or LPS and $V$. axillare for $6 \mathrm{~h}$. We selected this time point because the mRNA expression of the proinflammatory genes reached a peak at this point according to our previous studies [10]. As shown in Figure 4, after stimulation with LPS, the expression of IL-1 $\beta$,
IL-6, TNF- $\alpha$, MCP-1, and COX-2 was upregulated. Importantly, this upregulation was not observed in RAW264.7 cells cultured with $V$. axillare alone. Pretreatment with $V$. axillare $(250,500$, and $750 \mu \mathrm{g} / \mathrm{mL})$ significantly reduced the mRNA expression of these genes in a dose-dependent manner.

3.4. V. axillare Inhibits LPS-Induced Phosphorylation of $p 65$ and Prevented I $\kappa B-\alpha$ Degradation in RAW264.7 Macrophages. The genes repressed by $V$. axillare are normally controlled by the transcription factor NF- $\kappa$ B. Phosphorylation and degradation of $\mathrm{I} \kappa \mathrm{B}-\alpha$ lead to the phosphorylation of NF- $\kappa \mathrm{B}$ p 65 subunit. This phenomenon enables the release and translocation of the free NF- $\kappa \mathrm{B}$ into the nucleus, where it binds specifically to the $\kappa \mathrm{B}$ binding sites on DNA [11]. Therefore, 


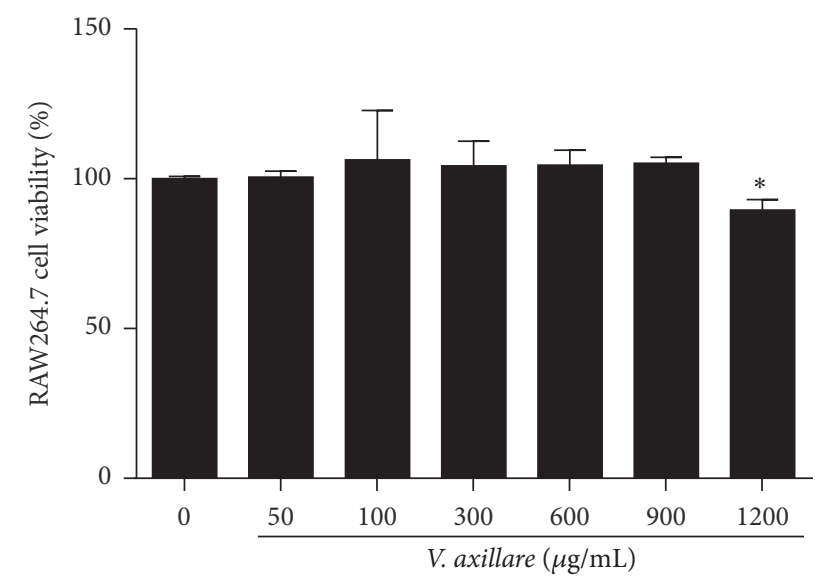

FIGURE 3: Effects of $V$. axillare on cell viability in RAW264.7 cells. Various concentrations of $V$. axillare were treated to the cells for $24 \mathrm{~h}$, and the cell viability was measured by CCK- 8 assay, as described in the Materials and Methods. The values represent the mean \pm SD from three independent experiments $\left({ }^{*} p<0.05\right.$ indicates a significant difference versus untreated control cells by Student's $t$ test).

we examined whether $V$. axillare altered these processes. As shown in Figure 5, LPS stimulation decreased the I $\kappa \mathrm{B}-\alpha$ protein level in untreated cells, which was consistent with the increased level of phosphorylated $\mathrm{p} 65$ and $\mathrm{I} \kappa \mathrm{B}-\alpha$. In contrast, pretreatment with $V$. axillare at 250,500 , and $750 \mu \mathrm{g} / \mathrm{mL}$ significantly reduced the phosphorylation of p65 and $\mathrm{I} \kappa \mathrm{B}$ $\alpha$ induced by LPS, indicating that $V$. axillare does exert an anti-inflammatory effect through the inhibition of LPSinduced $\mathrm{NF}-\kappa \mathrm{B}$ signaling pathway.

\section{Discussion}

In this study, we illustrated that, after pretreatment with $V$. axillare, the expression of proinflammatory cytokines (MCP1 , IFN- $\gamma$, TNF- $\alpha$, IL-6, and IL-12p70) in the serum of mice with LPS-induced ALI was notably reduced. We also found that $V$. axillare improved pulmonary histology in these mice. These findings collectively suggest that $V$. axillare may be effective for ALI prevention induced by LPS. In order to research the mechanisms of $V$. axillare action, we used RAW264.7 cells to evaluate changes in inflammatory mediators, cytokines, and signaling pathways.

During the progression of inflammation, proinflammatory cytokines such as TNF- $\alpha$ and IL- 6 are released by macrophages to protect the body from tissue injury or infection [12]. Furthermore, COX-2 is known as the rate-limiting enzyme that not only participates in the synthesis of prostaglandin E2, but also contributes to the inflammatory reaction [13]. The activation of macrophages leads to high expression level of MCP-1. The release of MCP-1 will cause the migration of tissue macrophages and blood monocytes to the site of inflammation, thereby promoting chronic inflammatory dysfunctions. Therefore, the mRNA expression levels of these inflammatory mediators are significant indicators during inflammation. In our study, the stimulation of LPS to the RAW264.7 cells led to considerable changes of several proinflammatory genes, including IL-1 $\beta$, IL-6, COX2, MCP-1, and TNF- $\alpha$, which correlate well with previous studies [14]. V. axillare also decreased the mRNA expression of these genes. These results provide direct evidence that $V$. axillare inhibited the development of inflammation through decreasing proinflammatory gene expressions in LPSstimulated macrophages.

LPS is a TLR4 agonist in macrophages [15]. The association between TLR4 and LPS results in the fast activation of NF- $\kappa \mathrm{B}[16]$. The expression of various cytokines, chemokines, cell-adhesion molecules, and growth factors in the lungs is closely regulated by the activation of NF- $\kappa \mathrm{B}$ [17]. Thus, the suppression of the proinflammatory mediators by the modulation of intracellular signaling in macrophages could be used for treating LPS-induced pulmonary diseases [18]. Our in vitro data suggest that NF- $\kappa \mathrm{B}$ was activated after LPS stimulation, evidenced by the degradation of $\mathrm{I} \kappa \mathrm{B}-\alpha$ and the phosphorylation of NF- $\kappa \mathrm{B}$ p 65 , which correlate well with previous findings $[6,14]$. These responses were prevented by $V$. axillare pretreatment. Similar results were acquired by immunohistochemical staining of less activated NF- $\kappa \mathrm{B}$ in vivo. These results suggest that the anti-inflammatory effect of $V$. axillare might be mediated via the inhibition of the NF$\kappa \mathrm{B}$ signaling pathways.

Macrophages play an important role during the innate immunity of the host response against many types of infections by producing various inflammatory mediators and adhesion molecules, which activate the immune system [19]. A large number of infection-associated disorders have been linked to the aberrant activation of macrophages, including ALI, sepsis, and septic shock. Therefore, agents which can inhibit the production/release of those inflammatory mediators can be developed as potential anti-inflammatory agents [20]. To further understand the underlying mechanisms of the anti-inflammatory effects of $V$. axillare, we evaluated the effects of $V$. axillare on the LPS-induced TLR4 signaling pathway in RAW264.7 cells. Previous mechanistic studies indicate that LPS-stimulated TLR4 activation was the most prominent mechanism of macrophage activation and can activate two different signaling pathways: the MyD88dependent and the TRIF-dependent pathways [21, 22]. The MyD88-dependent pathway plays a critical role in the regulation of macrophage activation, thereby promoting the activation of MAPKs and NF- $\kappa$ B. It is known that LPS-induced activation of MAPKs and NF- $\kappa$ B leads to the expression of several proinflammatory mediators in macrophages [23, 24]. We demonstrated that $V$. axillare extracts significantly suppressed LPS-induced phosphorylation of p65 and I $\kappa \mathrm{B}-\alpha$ in RAW264.7 macrophages, which was consistent with our in vivo data.

In terms of its chemical composition, the previous studies have found that $V$. axillare contains flavonoids, phenols, phenylpropanoids, terpenoids, tannins, and so forth [5]. Recently, three new phenylpropanoid glycosides isolated from $V$. axillare exerted strong anti-inflammatory effects, including procumboside $\mathrm{A}$. It is the major anti-inflammatory constituent of the EtOAc fraction of $V$. axillare. Interestingly, the anti-inflammatory effect of procumboside A seems to 


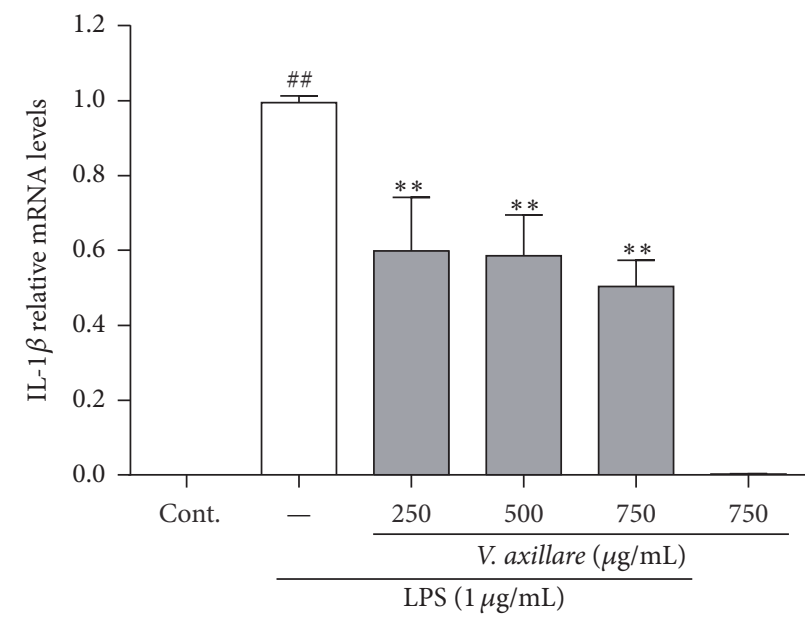

(a)

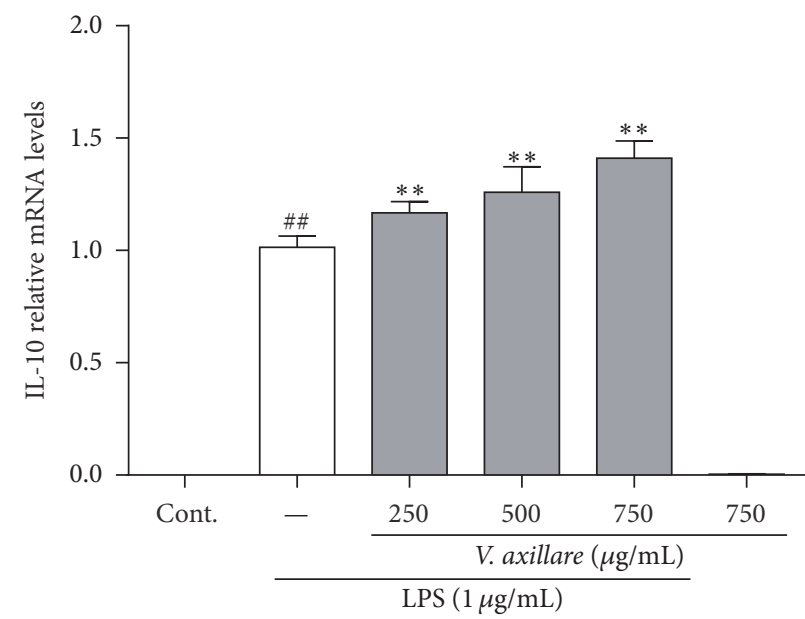

(c)

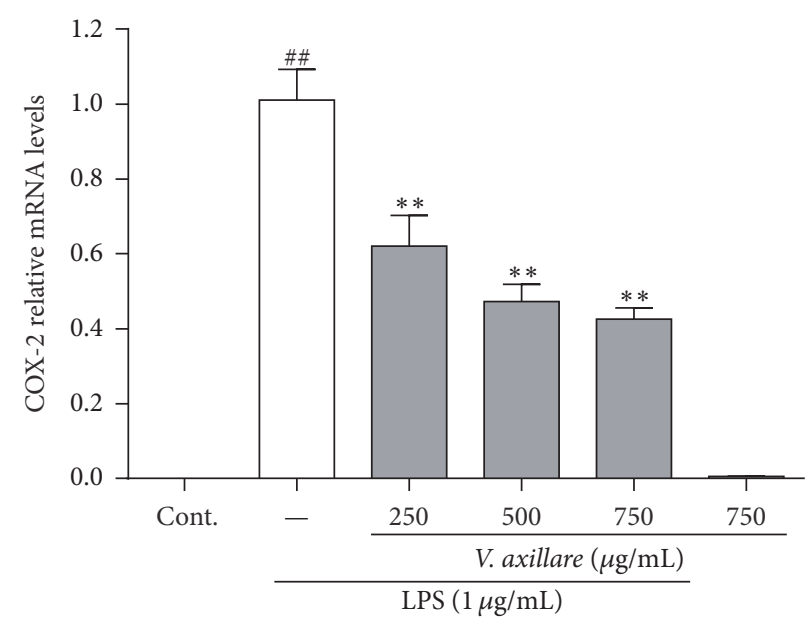

(e)

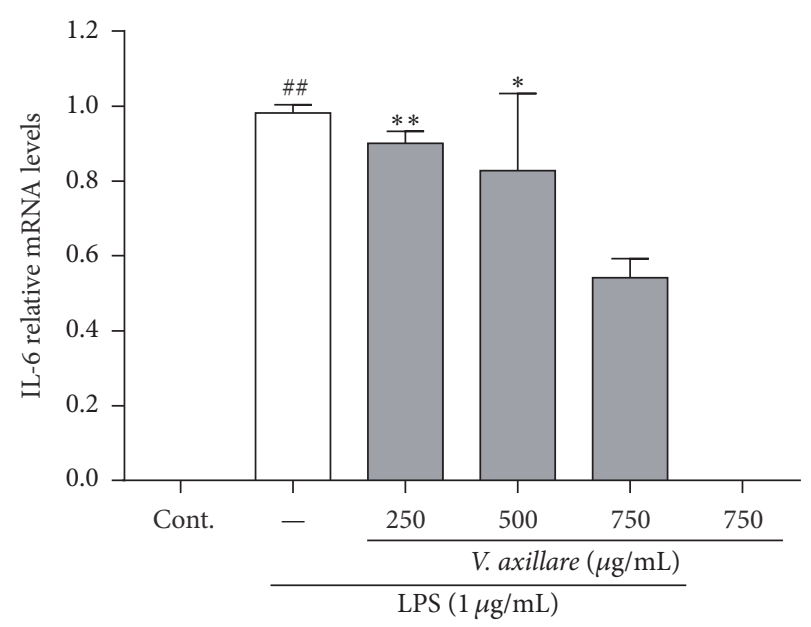

(b)

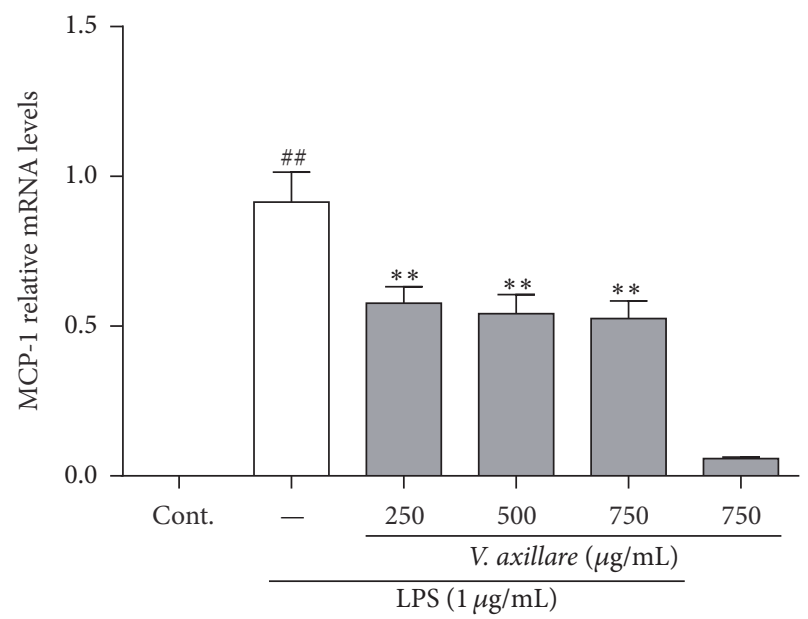

(d)

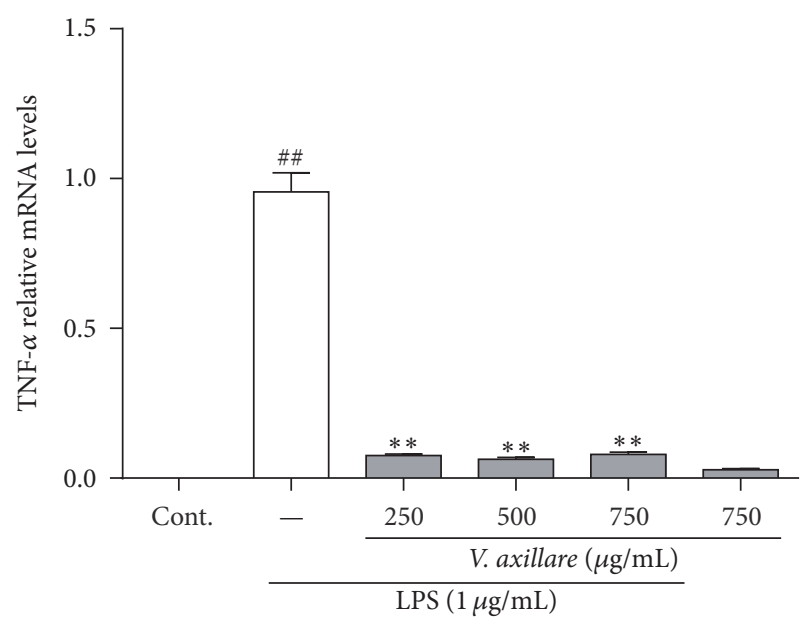

(f)

FIGURE 4: Effects of $V$. axillare on the gene expression of key proinflammatory genes in LPS-stimulated RAW264.7 cells. Various concentrations of $V$. axillare $(250,500$, and $750 \mu \mathrm{g} / \mathrm{mL})$ were treated to the cells for $1 \mathrm{~h}$ before incubation with LPS $(1 \mu \mathrm{g} / \mathrm{mL})$ for $6 \mathrm{~h}$. Additionally, one group was treated with $V$. axillare $(750 \mu \mathrm{g} / \mathrm{mL})$ alone to observe the effects of $V$. axillare on the RAW264.7 without LPS stimulation. The mRNA expression of (a) IL-1 $\beta$, (b) IL-6, (c) IL-10, (d) MCP-1, (e) COX-2, and (f) TNF- $\alpha$ was analyzed by qRT-PCR. Data are presented as the mean \pm SD from three independent experiments. Student's $t$-test was performed to compare individual groups $\left({ }^{*} p<0.05\right.$ and ${ }^{* *} p<0.01$ indicate a significant difference compared with the LPS group; ${ }^{\# \#} p<0.01$ indicates a significant difference compared with the untreated group). 


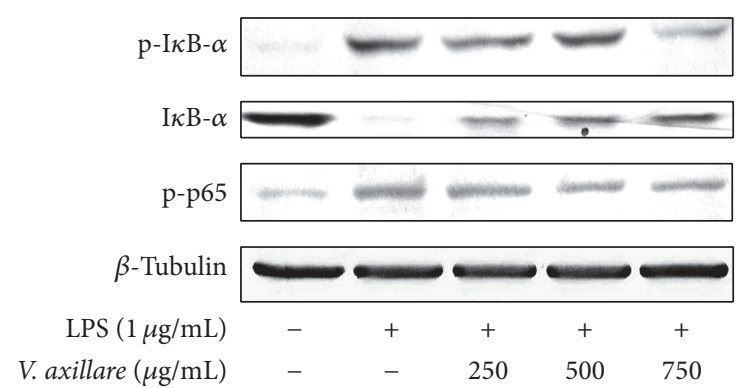

FIGURE 5: V. axillare inhibits LPS-induced phosphorylation of p65 and prevented I $\kappa$ B- $\alpha$ degradation in RAW264.7 macrophages. Cells were pretreated with $V$. axillare for $1 \mathrm{~h}$ before incubation with LPS for $30 \mathrm{~min}$. Levels of phosphorylated p65 and total and phosphorylated $\mathrm{I} \kappa \mathrm{B}-\alpha$ were determined by western blot analysis. $\beta$-Tubulin was used as a loading control.

be stronger than that of dexamethasone which is also used as a positive drug in a previous study [7]. Nevertheless, the interactions among active compounds in the natural products are complex with potential synergistic/antagonistic effects, which attracts great interest in TCM research. Hence, it is valuable to study the interaction among the aforementioned compounds in V. axillare in the future.

Taken together, our study confirmed that the aqueous extract of $V$. axillare exerted strong anti-inflammatory effects by attenuating LPS-induced lung injury. V. axillare prevented the overproduction of inflammatory cytokines in ALI mice. Histological analysis indicated that $V$. axillare significantly improved lung injury and decreased pulmonic NF- $\kappa$ B activation. We also noticed that $V$. axillare could downregulate the mRNA expressions of IL- $1 \beta$, IL-6, IL-10, MCP-1, COX-2, and TNF- $\alpha$ in LPS-activated RAW264.7 cells. These effects seem to be mediated, at least in part, by the inhibition of $\mathrm{NF}-\kappa \mathrm{B}$ activation, which provides first-hand evidence for the development of anti-inflammatory drugs in the future. Further studies about the active components in $V$. axillare are required for better understanding the protective effects against ALI.

\section{Competing Interests}

The authors declare that they have no competing interests.

\section{Acknowledgments}

The authors are grateful to Yefeng Lu from the College of Yangzhou University for providing technical assistance. This work was supported by Zhejiang Provincial Program for the Cultivation of High-Level Innovative Health Talents to Minli Chen and the Natural Science Foundation of Zhejiang Province (no. LY14H280006).

\section{References}

[1] J. Grommes and O. Soehnlein, "Contribution of neutrophils to acute lung injury," Molecular Medicine, vol. 17, no. 3-4, pp. 293307, 2011.
[2] Z.-Q. Su, Z.-Z. Mo, J.-B. Liao et al., "Usnic acid protects LPS-induced acute lung injury in mice through attenuating inflammatory responses and oxidative stress," International Immunopharmacology, vol. 22, no. 2, pp. 371-378, 2014.

[3] R. Herrero, R. Guillamat, L. Chimenti et al., "A novel and sustained acute lung injury model induced by hydrochloric acid and LPS in rats," American Journal of Respiratory Critical Care Medicine, vol. 189, p. A4986, 2014.

[4] D. S. El-Agamy, "Nilotinib ameliorates lipopolysaccharide-induced acute lung injury in rats," Toxicology and Applied Pharmacology, vol. 253, no. 2, pp. 153-160, 2011.

[5] X.-H. Deng, F.-F. Fang, C.-J. Zheng, Y. Wu, and L.-P. Qin, "Monoterpenoids from the whole herb of Veronicastrum axillare," Pharmaceutical Biology, vol. 52, no. 5, pp. 661-663, 2014.

[6] Y. Du, W. Zhao, L. Lu et al., "Study on the antiulcer effects of Veronicastrum axillare on gastric ulcer in rats induced by ethanol based on tumor necrosis factor- $\alpha$ (TNF- $\alpha$ ) and endothelin-1 (ET-1)," Asian Pacific Journal of Tropical Biomedicine, vol. 3, no. 12, pp. 925-930, 2013.

[7] C.-J. Zheng, X.-H. Deng, Y. Wu, Y.-P. Jiang, J.-Y. Zhu, and L.P. Qin, "Antiinflammatory effects and chemical constituents of Veronicastrum axillare," Phytotherapy Research, vol. 28, no. 10, pp. 1561-1566, 2014.

[8] J.-Y. Xie, H.-Y. Di, H. Li, X.-Q. Cheng, Y.-Y. Zhang, and D.F. Chen, "Bupleurum chinense DC polysaccharides attenuates lipopolysaccharide-induced acute lung injury in mice," Phytomedicine, vol. 19, no. 2, pp. 130-137, 2012.

[9] H.-Y. Lin, Y. Zhao, J.-N. Yu, W.-W. Jiang, and X.-L. Sun, "Effects of traditional Chinese medicine Wei-Wei-Kang-Granule on the expression of EGFR and NF-KB in chronic atrophic gastritis rats," African Journal of Traditional, Complementary, and Alternative Medicines, vol. 9, no. 1, pp. 1-7, 2012.

[10] K. Wang, S. Ping, S. Huang et al., "Molecular mechanisms underlying the in vitro anti-inflammatory effects of a flavonoidrich ethanol extract from chinese propolis (poplar type)," Evidence-Based Complementary and Alternative Medicine, vol. 2013, Article ID 127672, 11 pages, 2013.

[11] T. Berger, M. E. Saunders, and T. W. Mak, "Dissection of signaling in inflammation: three novel inflammatory regulators," Cold Spring Harbor Symposia on Quantitative Biology, vol. 78, no. 1, pp. 141-147, 2013.

[12] M. Gleeson, N. C. Bishop, D. J. Stensel, M. R. Lindley, S. S. Mastana, and M. A. Nimmo, "The anti-inflammatory effects of exercise: mechanisms and implications for the prevention and treatment of disease," Nature Reviews Immunology, vol. 11, no. 9, pp. 607-615, 2011.

[13] S. Bhattacharjee, A. Bhattacharjee, S. Majumder, S. B. Majumdar, and S. Majumdar, "Glycyrrhizic acid suppresses Cox2-mediated anti-inflammatory responses during Leishmania donovani infection," Journal of Antimicrobial Chemotherapy, vol. 67, no. 8, Article ID dks159, pp. 1905-1914, 2012.

[14] K. Wang, L. Hu, X.-L. Jin et al., "Polyphenol-rich propolis extracts from China and Brazil exert anti-inflammatory effects by modulating ubiquitination of TRAF6 during the activation of NF- $\kappa$ B," Journal of Functional Foods, vol. 19, pp. 464-478, 2015.

[15] X. Xu, P. Yin, C. Wan et al., "Punicalagin inhibits inflammation in LPS-induced RAW264.7 macrophages via the suppression of TLR4-mediated MAPKs and NF- $\kappa$ B activation," Inflammation, vol. 37, no. 3, pp. 956-965, 2014.

[16] A. Remppis, F. Bea, H. J. Greten et al., "Rhizoma coptidis inhibits LPS-induced MCP-1/CCL2 production in murine macrophages 
via an AP-1 and NF B-dependent pathway," Mediators of Inflammation, vol. 2010, Article ID 194896, 8 pages, 2010.

[17] Y. Zhao, I. A. Gorshkova, E. Berdyshev et al., "Protection of LPSinduced murine acute lung injury by sphingosine-1-phosphate lyase suppression," American Journal of Respiratory Cell and Molecular Biology, vol. 45, no. 2, pp. 426-435, 2011.

[18] T. Nojiri, H. Hosoda, T. Tokudome et al., "Atrial natriuretic peptide inhibits lipopolysaccharide-induced acute lung injury," Pulmonary Pharmacology and Therapeutics, vol. 29, no. 1, pp. 24-30, 2014.

[19] M. F. Linton and S. Fazio, "Macrophages, inflammation, and atherosclerosis," International Journal of Obesity, vol. 27, no. 3, pp. S35-S40, 2003.

[20] B. Maity, S. K. Yadav, B. S. Patro, M. Tyagi, S. K. Bandyopadhyay, and S. Chattopadhyay, "Molecular mechanism of the anti-inflammatory activity of a natural diarylnonanoid, malabaricone C," Free Radical Biology and Medicine, vol. 52, no. 9, pp. 16801691, 2012.

[21] M.-Z. Xia, Y.-L. Liang, H. Wang et al., "Melatonin modulates TLR4-mediated inflammatory genes through MyD88and TRIF-dependent signaling pathways in lipopolysaccharidestimulated RAW264.7 cells," Journal of Pineal Research, vol. 53, no. 4, pp. 325-334, 2012.

[22] H. N. Jnawali, E. Lee, K.-W. Jeong, A. Shin, Y.-S. Heo, and Y. Kim, "Anti-inflammatory Activity of Rhamnetin and a model of its binding to c-Jun NH2-terminal kinase 1 and p38 MAPK," Journal of Natural Products, vol. 77, no. 2, pp. 258-263, 2014.

[23] A. Haque, N. Koide, I. Iftakhar-E-Khuda et al., "Flavopiridol inhibits lipopolysaccharide-induced TNF- $\alpha$ production through inactivation of nuclear factor- $\kappa \mathrm{B}$ and mitogen-activated protein kinases in the MyD88-dependent pathway," Microbiology and Immunology, vol. 55, no. 3, pp. 160-167, 2011.

[24] X. Chen, X. Yang, T. Liu et al., "Kaempferol regulates MAPKs and NF- $\kappa \mathrm{B}$ signaling pathways to attenuate LPS-induced acute lung injury in mice," International Immunopharmacology, vol. 14, no. 2, pp. 209-216, 2012. 


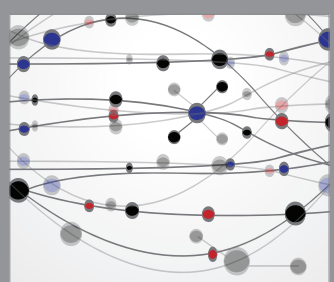

The Scientific World Journal
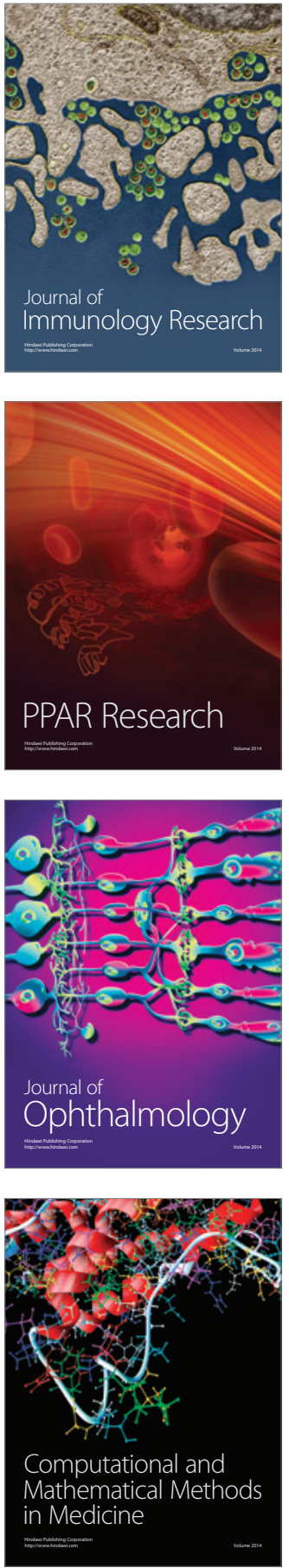

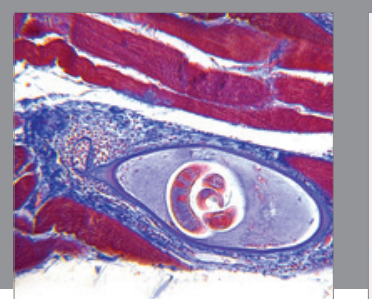

Gastroenterology Research and Practice

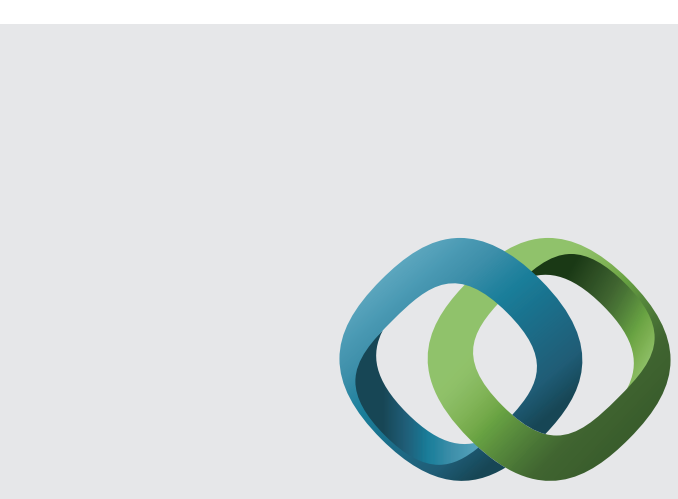

\section{Hindawi}

Submit your manuscripts at

http://www.hindawi.com
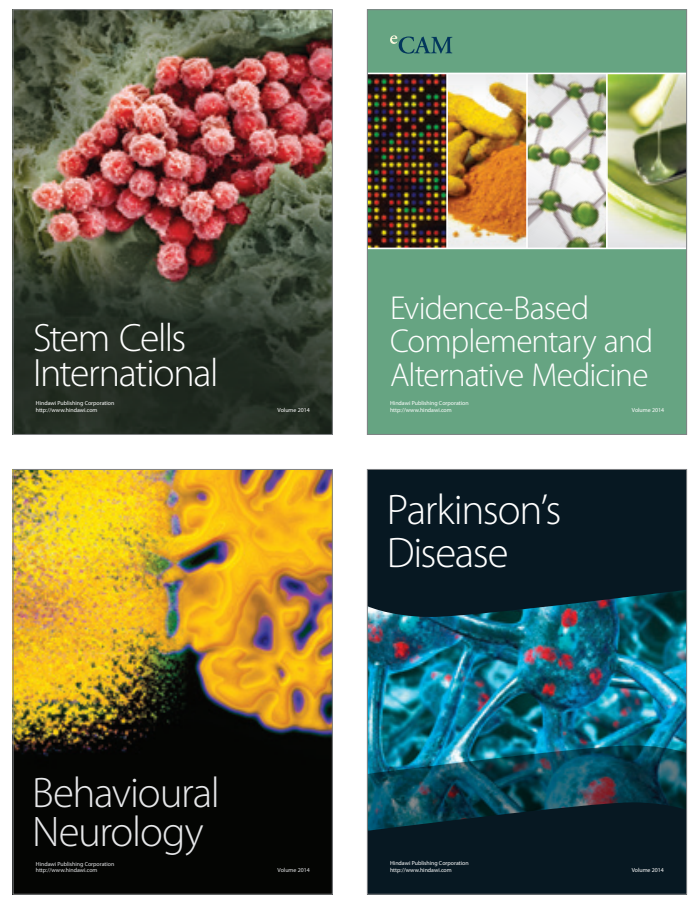
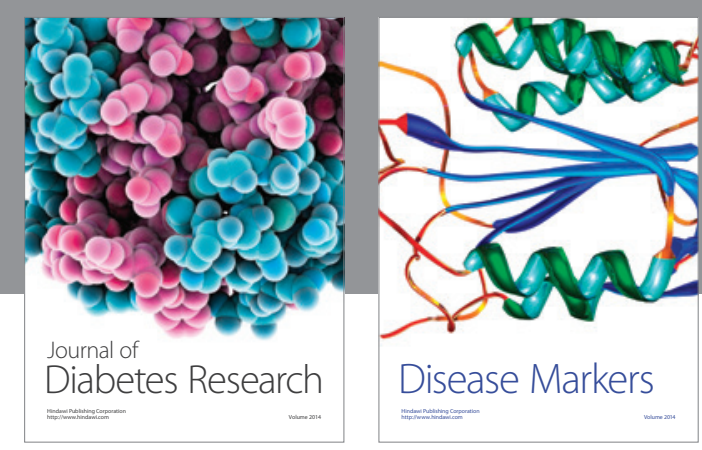

Disease Markers
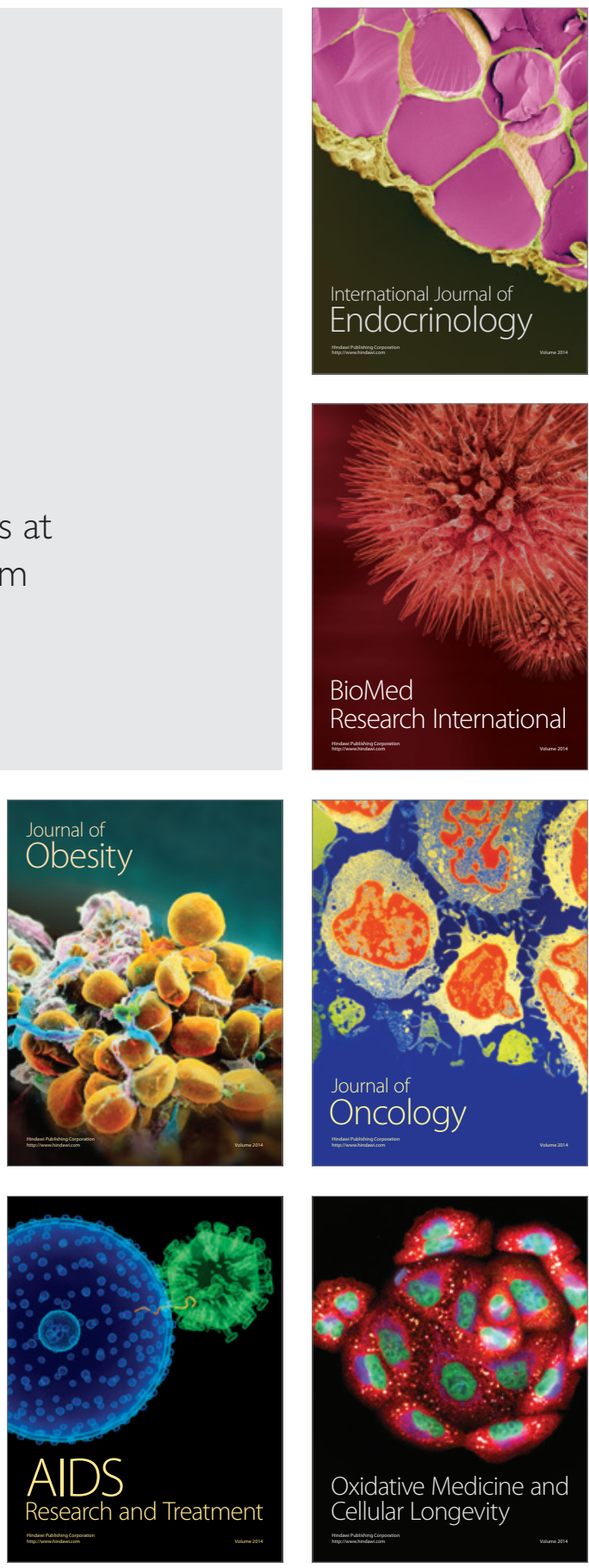